\title{
Use of an Internal Retractor for Percutaneous Full- Endoscopic Resection in Cervical Intervertebral Disc Herniation with a Posterior Approach
}

\author{
Shu Nakamura, Mitsuto Taguchi \\ Department of Orthopedic Surgery, Aichi Spine Hospital, Inuyama, Japan
}

\begin{abstract}
Study Design: A preliminary case series study.
Purpose: For the safety of performing a posterior percutaneous full-endoscopic cervical discectomy.

Overview of Literature: Because of the lack of space for inserting an outer sheath above the intervertebral disc in the spinal canal, grasping the herniated disc with forceps while retracting the nerves with the forceps itself was required. This procedure produces the risk of inadvertently injuring nerves because of inadequate visualization of the hernia and inadequate protection of the nerve.

Methods: Our new internal retractor can be inserted into the working channel of a percutaneous full-endoscope, enabling the insertion of a second tool. After partial foraminotomy, the internal retractor and forceps were manipulated to reliably retract the nerve root. Finally, the herniated disc was resected under an endoscopic view.

Results: All six cases had a good postoperative course, and postoperative neuropathy was not observed.

Conclusions: This internal retractor allows for the secure resection herniated cervical intervertebral discs.
\end{abstract}

Keywords: Spine; Herniated disc; Discectomy; Percutaneous

\section{Introduction}

The resection of a herniated cervical intervertebral disc is often performed using an anterior approach. However, there are problems associated with anterior cervical discectomy and fusion [1], including approach-related complications or the degeneration of adjacent segments [2-5]. The discs are inevitably damaged during anterior access surgery even while using a percutaneous full-endoscope $(\mathrm{PE})$. Also, a reduction of disc height by $11.2 \%-23 \%$ has been observed after this procedure [6-8]. Even with a keyhole corpectomy, the vertebral body edge or coronoid process can be injured markedly, resulting in reduced rigidity during lateral flexion or rotation [9-11] and loss of the protective wall of foramen against disc herniation. These events may be avoided by using a posterior approach, but this approach requires great care to avoid nerve injury while reaching the herniation.

Although PE surgery is minimally invasive, the diameter of the PE is small, and the diameter of its working channel is even smaller. Therefore, the working channel only allows for the insertion of only one tool at a time,

Received May 2, 2019; Revised Aug 29, 2019; Accepted Sep 19, 2019

Corresponding author: Shu Nakamura

Department of Orthopedic Surgery, Aichi Spine Hospital, 31-1 Kamiike, Goroumaru, Inuyama-shi, Aichi, 484-0066, Japan

Tel: +81-568-20-9100, Fax: +81-568-20-9107, E-mail: nakamura@itoortho.or.jp 
and it requires the surgeon to manipulate with one inserted tool while securing the working space using the outer sheath outside of the endoscope. Unlike the lumbar spine, the cervical spine has very little free space within the spinal canal and the intervertebral foramen occupied by the spinal cord, dura mater, and nerve root. Therefore, it is dangerous to insert a 7- to 8 -mm-diameter outer sheath into this space. In clinical practice, the outer sheath is inserted on the vertebral bone before the spinal canal. When viewed posteriorly, the spinal cord, nerve root, and surrounding soft tissue are located over the disc, thus limiting the visual field during the resection. Therefore, using forceps almost blindly to resect the herniated disc while also retracting the nerve with forceps is required, which poses a risk of nerve/vessel injury. Additionally, epidural bleeding is also possible during this procedure. Therefore, it is necessary to establish how to secure an adequate visual field while protecting the nerve during this operation.

We recently devised a new tool that may be used during the percutaneous full-endoscopic resection of the herniated cervical disc using a posterior approach [12]. Using this tool, we successfully resected the herniated cervical discs in a safer manner than before.

\section{Materials and Methods}

\section{Indication}

This operation was indicated for patients with radiculopathy whose magnetic resonance imaging (MRI) scans revealed cervical disc herniation in the lateral part of the spinal canal to the intervertebral foramen and who were dissatisfied with conservative treatment. Patients with median-type or ossified disc herniation were excluded from this study. Patients who underwent this operation after September 2017 and had been followed up for 1 year or more since the operation were included in the study.

\section{Surgical method}

Before the operation, indigo carmine was injected anteriorly into the intervertebral disc with the patient in the supine position under general anesthesia; this manipulation is not indispensable. The patient was placed in the prone position using a Hall frame and a ProneView (Dupaco, Oceanside, CA, USA). The head was fixed with tape while the patient's neck was slightly flexed. A C-arm fluoroscope was set so that the frontal and lateral views of the cervical spine could be provided without a limit, and the patient was continuously monitored using free-run electromyography. The surgeon made a 7-mm incision in the paramedian area of the affected side. An 8-mm-diameter outer sheath and a PE (asap endoscopic products, Umkirch, Germany) were inserted using a serial dilator on the vertebral arch of the affected side. Bone fenestration behind the herniated disc was conducted using a diamond bur. After the ligamentum flavum was resected partially and the nerve root and dura mater confirmed, a new internal retractor, which will be described in this manuscript, was inserted into the working channel of the PE. The internal retractor, which was a cylinder with an outer diameter of 4 $\mathrm{mm}$, had a tip of the quadrant and long beak. A tool with an outer diameter of $2.5 \mathrm{~mm}$ could be inserted into its inner channel (Figs. 1,2). While the nerve root and dura mater was retracted with the beak of this internal retractor, the open side of the retractor tip was directed toward

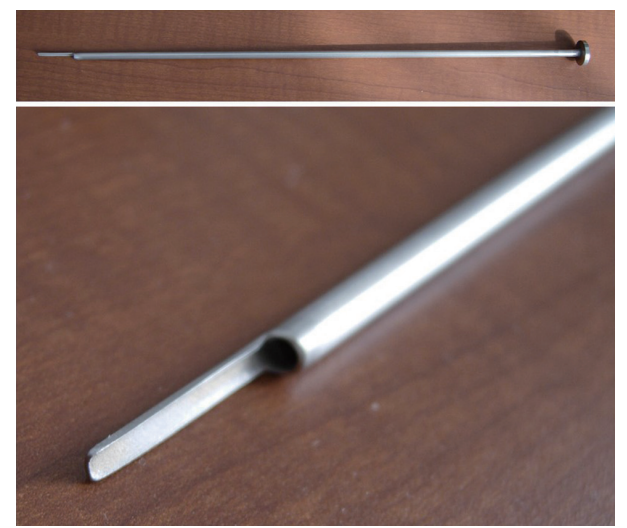

Fig. 1. Internal retractor. A cylinder with an outer diameter of $4 \mathrm{~mm}$ had a tip of quadrant beak.

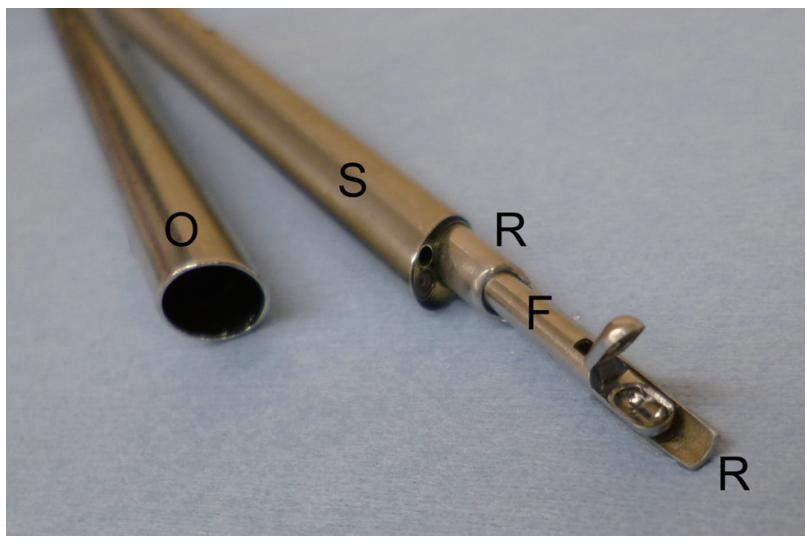

Fig. 2. Internal retractor and percutaneous full-endoscope. R, internal retractor; 0, 8-mm outer sheath; S, percutaneous full-endoscope; F, 2.5-mm forceps. 

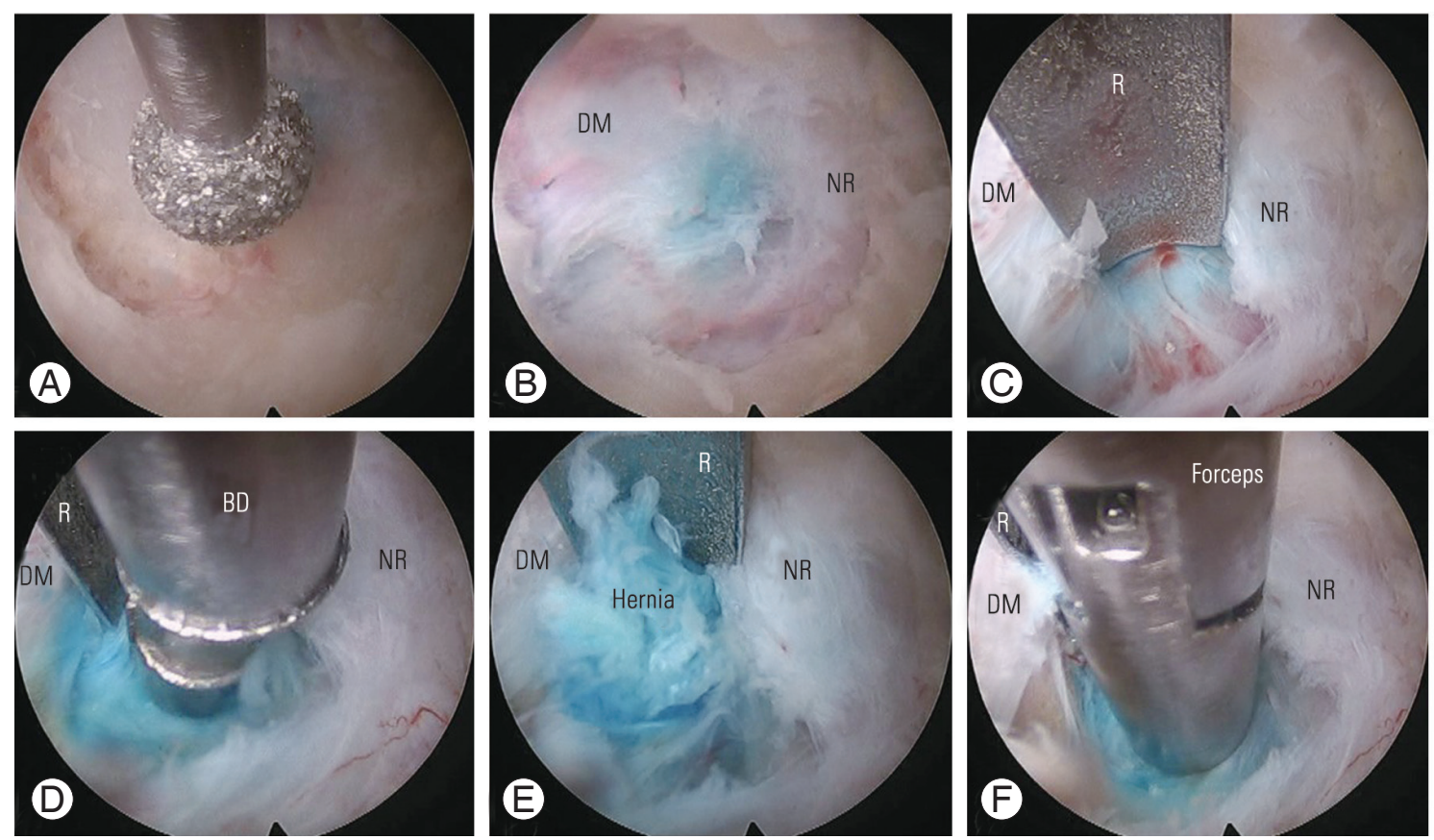

Fig. 3. Intraoperative endoscopic images. The cranial side is on the right, whereas the lateral side is at the bottom of the images. (A) Laminotomy was conducted using a diamond bur. (B) The ligamentum flavum was partially resected, and the NR and DM were confirmed. (C) An R was inserted into the working channel of the percutaneous full-endoscope to retract the NR and DM. In this case, there was no prolapsing mass of herniation. (D) The superficial layer of the hernia was crushed using a radiofrequency BD. (E) The surrounding tissue was compressed using the tip of the $R$ and the hernia was extruded. (F) 2.5-mm forceps was inserted into the $R$, and the hernia was resected while retracting the NR and DM with the R. NR, nerve root; $\mathrm{DM}$, dura mater; $\mathrm{R}$, internal retractor; $\mathrm{BD}$, bipolar device.

the lens of the endoscope, and the herniated disc was resected under an endoscopic view using 2.5 -mm forceps inserted into the internal retractor (Figs. 3, 4). In cases where there was no prolapsing mass of herniation, the superficial layer of the herniated disc was crushed using the tip of the internal retractor or others, and the surrounding tissue was compressed so that the herniated disc could be extruded. Epidural bleeding, if any, was stopped using a radiofrequency bipolar device (Elliquence, Baldwin, NY, USA) that was inserted into the internal retractor while retracting the nerves.

The patient was allowed to ambulate 3 hours postoperatively. During the following day, the drain was removed, and the patient was discharged. The patient was required to wear a soft neck collar for 1 week.

\section{Evaluation}

The symptoms of the neck and arms were rated on a Visual Analog Scale (VAS, 10-0) using a self-administered questionnaire. The degree of patient satisfaction was rated according to a four-category scale (excellent, good, fair, or poor).

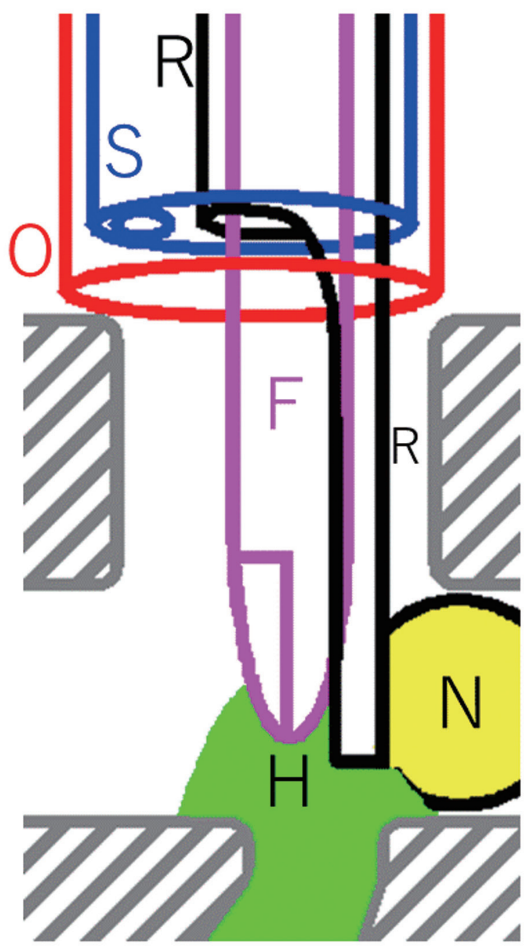

Fig. 4. A schematic illustration of a transmission image depicting the intraoperative device location. $0,8-\mathrm{mm}$ outer sheath; $\mathrm{S}$, percutaneous full-endoscope; $\mathrm{R}$, internal retractor; F, 2.5-mm forceps; $H$, herniated disc; $N$, nerve root. 


\section{Ethical statement}

This study was approved (no. IR18301) by our institutional review board. Informed consent was obtained from all individual participants included in the study.

\section{Results}

\section{Operation results}

This operation was performed in six patients (five men and one woman; mean age, 53.0 years; mean operative time, 70.3 minutes; operated spine level, C5-6 in one case and C6-7 in five cases). In all cases, the herniated disc was resected successfully while securing the visual field, and no operation-related neuropathy occurred. The mean follow-up period was 16.3 months. The VAS score (0-10) for neck symptoms decreased from 6.0 (preoperative average) to 2.0 (final average), whereas the VAS score for arm symptoms decreased from 6.3 (preoperative average) to 1.3 (final average). The degree of patient satisfaction was excellent for five cases and good for one case.

\section{Representative case}

One patient complained of pain from the right side of

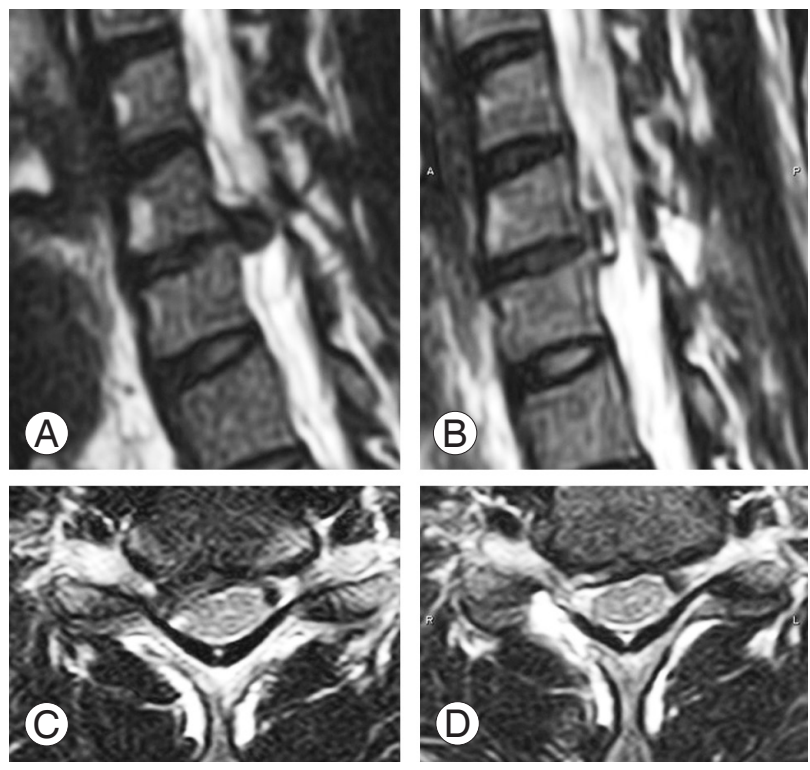

Fig. 5. Magnetic resonance imaging scan of the representative case. A large herniated disc on the right side of the spinal canal at the $C 5 / 6$ level was resected sufficiently. (A) Preoperative sagittal view. (B) Postoperative sagittal view. (C) Preoperative axial view. (D) Postoperative axial view. the neck to the right arm and a feeling of numbness in the first to third fingers of the right hand. MRI revealed a large herniated disc on the right side of the spinal canal at the C5/6 level. Pain was alleviated postoperatively (Figs. 3, 5).

\section{Discussion}

During PE surgery, the visual field is secured with an outer sheath. The cervical spine has too little free space within the spinal canal and the intervertebral foramen to insert a 7 - to 8 -mm-diameter outer sheath through this space. If an ostectomy is performed to safely insert the outer sheath, the pedicle needs to be almost completely resected, and this is unrealistic. Therefore, the insertion of an outer sheath to the intervertebral disc has been virtually impossible using a posterior approach because of the risk of the neural damage during the operation.

Anatomically, the nerve root and perineural soft tissue are located dorsal to the cervical disc [13], which limits the visual field during posterior resection of the herniated disc. Additionally, when bleeding from the epidural venous plexus occurs, it is difficult to stop and makes the visual field worse. Inadequate visualization of the hernia and inadequate protection of the nerve also create the risk of injuring the nerves and blood vessels. For instance, one prior case of the arterial injury that occurred at our hospital required open conversion.

Using conventional PE, it was impossible to simultaneously insert two tools into its inner channel. Hence, we devised a tool to enable such insertion. By using the retractor, we avoid injury to the nerves and we were able to safely stop bleeding using a radiofrequency bipolar device. This tool will be also useful for performing on the upperlevel lumbar vertebrae.

Even when only decompression is performed via posterior ostectomy without resection of the herniated disc, the patient's condition may improve considerably $[14,15]$. However, an excessive amount of bone needs to be resected in cases of wide disc herniation. Furthermore, if the herniated disc is left unresected, it may produce an inflammatory response. Moreover, the site of cervical disc herniation is likely to undergo ossification [16], which leads to permanent elevation. This may increase the risk of restenosis because the resected vertebral arch subsequently undergoes active bone regeneration [17].

Another design that may be adopted instead of this tool 
is a long, narrow, plate-formed retractor. However, rigidity will be insufficient with this form. An outer sheath with a considerably narrow beak is possible to create. Nevertheless, such a retractor involves problems such as the necessity of intraoperative outer sheath exchange, difficulties in delicate manipulation due to the surrounding tissue restricting the motion of the outer sheath, and invasion of muscle and other structures into the visual field while advancing and retreating the outer sheath. Our new tool avoids these problems. It is desirable to use an outer sheath in order to retract muscles above the vertebral arch, whereas another tool can be used to perform delicate manipulation to avoid nerve injury within the spinal canal (Fig. 4). With our technique, three tools (including the outer sheath) are manipulated, and each of these tools is applied appropriately to each layer of the area of operation.

Intraoperative retraction of nerves should carefully be performed using an electromyographic monitor and prioritizing sufficient osseous decompression rather than forcefully attempting total resection of the hernia.

This study did not include many cases, and the postoperative follow-up period was short. We will further evaluate the utility and risks associated with this technique in a larger number of cases.

\section{Conclusions}

Posterior percutaneous full-endoscopic cervical discectomy using an internal retractor enables safer resection of the herniated disc by manipulating two tools within the inner working channel of the PE.

\section{Conflict of Interest}

No potential conflict of interest relevant to this article was reported.

\section{ORCID}

Shu Nakamura: https://orcid.org/0000-0002-8015-7571

\section{References}

1. Smith GW, Robinson RA. The treatment of certain cervical-spine disorders by anterior removal of the intervertebral disc and interbody fusion. J Bone Joint
Surg Am 1958;40-A:607-24.

2. Hilibrand AS, Carlson GD, Palumbo MA, Jones PK, Bohlman HH. Radiculopathy and myelopathy at segments adjacent to the site of a previous anterior cervical arthrodesis. J Bone Joint Surg Am 1999;81:51928.

3. Matsumoto M, Okada E, Ichihara D, et al. Anterior cervical decompression and fusion accelerates adjacent segment degeneration: comparison with asymptomatic volunteers in a ten-year magnetic resonance imaging follow-up study. Spine (Phila Pa 1976) 2010;35:36-43.

4. Baba H, Furusawa N, Imura S, Kawahara N, Tsuchiya $\mathrm{H}$, Tomita $\mathrm{K}$. Late radiographic findings after anterior cervical fusion for spondylotic myeloradiculopathy. Spine (Phila Pa 1976) 1993;18:2167-73.

5. Lopez-Espina CG, Amirouche F, Havalad V. Multilevel cervical fusion and its effect on disc degeneration and osteophyte formation. Spine (Phila Pa 1976) 2006;31:972-8.

6. Ahn Y, Lee SH, Shin SW. Percutaneous endoscopic cervical discectomy: clinical outcome and radiographic changes. Photomed Laser Surg 2005;23:3628.

7. Ruetten S, Komp M, Merk H, Godolias G. Full-endoscopic anterior decompression versus conventional anterior decompression and fusion in cervical disc herniations. Int Orthop 2009;33:1677-82.

8. Nakamura S, Taguchi M. Percutaneous endoscopic cervical discectomy: surgical approaches and postoperative imaging changes. Asian Spine J 2018;12:2949.

9. Chen TY, Crawford NR, Sonntag VK, Dickman CA. Biomechanical effects of progressive anterior cervical decompression. Spine (Phila Pa 1976) 2001;26:6-14.

10. Wang Z, Zhao H, Liu JM, Tan LW, Liu P, Zhao JH. Resection or degeneration of uncovertebral joints altered the segmental kinematics and load-sharing pattern of subaxial cervical spine: a biomechanical investigation using a C2-T1 finite element model. J Biomech 2016;49:2854-62.

11. Clausen JD, Goel VK, Traynelis VC, Scifert J. Uncinate processes and Luschka joints influence the biomechanics of the cervical spine: quantification using a finite element model of the C5-C6 segment. J Orthop Res 1997;15:342-7.

12. Ruetten S, Komp M, Merk H, Godolias G. A new 
full-endoscopic technique for cervical posterior foraminotomy in the treatment of lateral disc herniations using 6.9-mm endoscopes: prospective 2-year results of 87 patients. Minim Invasive Neurosurg 2007;50:219-26.

13. Tanaka N, Fujimoto Y, An HS, Ikuta Y, Yasuda M. The anatomic relation among the nerve roots, intervertebral foramina, and intervertebral discs of the cervical spine. Spine (Phila Pa 1976) 2000;25:286-91.

14. Yagi S. Microendoscopic posterior cervical foraminotomy for cervical disc herniation using METRx system. J West Jpn Res Soc Spine 2006;32:121-4.
15. Tagami A, Baba H, Hiura T, et al. Surgical outcomes of the microendoscopic cervical key hole foraminotomy without discectomy. J Spine Res 2010;1:1194-7.

16. Ida Y, Morimoto T, Tei R, Inui T, Nakase H, Sakaki T. Immunolocalization of growth factor in degeneration of cervical intervertebral discs. Spinal Surg 2002;16:21-8.

17. Nakamura S, Taguchi M. Area of ostectomy in posterior percutaneous endoscopic cervical foraminotomy: images and mid-term outcomes. Asian Spine J 2017;11:968-74. 